\title{
Conjugate root offset-QAM for orthogonal multicarrier transmission
}

\author{
Maximilian Matthé* and Gerhard Fettweis
}

\begin{abstract}
Current implementations of orthogonal frequency-division multiplexing (OFDM)/offset quadrature amplitude modulation (OQAM) are restricted to band-limited symmetric filters. To circumvent this, non-symmetric conjugate root (CR) filters are proposed for OQAM modulation. The idea is applied to Generalized Frequency Division Multiplexing (GFDM) and a method for achieving transmit diversity with OQAM modulation is presented. The proposal reduces implementation complexity compared to standard OQAM by modifying the transmit phase space which is exploited by a real-valued fast Fourier transform (FFT) operation. Generalized frequency division multiplexing (GFDM)/CR-OQAM outperforms conventional GFDM using QAM modulation in terms of symbol error rate in fading multipath channels and provides a more localized spectrum compared to GFDM/OQAM.
\end{abstract}

Keywords: Quadrature amplitude modulation, Multicarrier modulation, Space-time coding

\section{Introduction}

With 5G on the horizon, new waveforms for the PHY layer are investigated that are suitable for the upcoming requirements [1]. In particular, good time-frequency localization (TFL) of the transmit signal is required to cope with asynchronicities [2] and to provide a low outof-band (OOB) radiation, which is needed for spectral agility and carrier aggregation. Filtered multicarrier (MC) systems [3] provide the means for good TFL by adaptation of the prototype filter, and spectral agility is achieved by switching on and off certain subcarriers. High spectral efficiency is important to serve the increased demand for high-speed data access [1]. Hence, a future waveform should transmit symbols at the Nyquist rate in order to not waste valuable time-frequency resources.

Even in a distortion-free channel, the Balian-Low theorem (BLT) prohibits the distortion-free reconstruction of complex valued symbols sent at Nyquist rate when using filters with good TFL [4]. Hence, in terms of symbol error rate (SER), QAM MC systems with good TFL perform worse than orthogonal systems without good TFL. One possibility to circumvent the BLT is to transmit real-valued symbols at twice the Nyquist rate, which was initially proposed in [3]. This gave rise

*Correspondence: maximilian.matthe@ifn.et.tu-dresden.de Vodafone Chair Mobile Communications Systems, Technical University Dresden, Dresden, Germany to the well-known orthogonal frequency-division multiplexing (OFDM)/offset quadrature amplitude modulation (OQAM) [5] MC system where both orthogonality and good TFL is kept by using OQAM, which is proposed for upcoming $5 \mathrm{G}$ networks [1].

In the current OQAM proposals, the employed realvalued prototype filter is required to be bandlimited and symmetric and a phase shift of $\frac{\pi}{2}$ is maintained between adjacent subcarriers. In [6], low-complexity implementations of these schemes are described and also, a method is proposed to relax the requirement of symmetry of the employed prototype filter which is advantageous since it is shown in [7] that non-symmetric filters can be more robust against multipath fading. However, the approach presented in [6] suffers from an increased implementation complexity compared to conventional OQAM since it requires to use two poly-phase networks (PPNs).

In this paper we contribute another version of $\mathrm{MC}$ OQAM modulation, named conjugate root (CR) OQAM (CR-OQAM), which allows to use the class of nonsymmetric CR filters, removing the $\frac{\pi}{2}$ phase shift. We show that the new proposal can be implemented with fewer arithmetic operations than the conventional OFDM/OQAM system analyzed in [6]. We further apply CR-OQAM to generalized frequency division multiplexing (GFDM) [8], which is another 5G candidate waveform that normally uses QAM modulation and hence 
suffers from the BLT. With CR-OQAM, we provide a welllocalized orthogonal system, improving the SER performance in fading multipath channels compared to standard GFDM. We provide analysis regarding spectral and peakto-average power ratio (PAPR) properties for this new waveform and compare them with the existing systems. Additionally, time-reversal space-time coding (TR-STC) [9] is applied to GFDM/CR-OQAM to exploit multiantenna diversity, showing that in GFDM, STC can be easily combined with OQAM modulation. For the timecontinuous OFDM/OQAM, several methods for application of STC have been proposed. For example in [10], the authors suggest to achieve transmit diversity by adopting a CDMA scheme. However, the scheme requires the channel to be flat over a range of subcarriers which is especially hard to achieve in rich-scattering environments. Furthermore, the authors in [11] propose to artificially divide the OFDM/OQAM stream into blocks that are separated by guard intervals. The data in each block is then space-time encoded by reversing the time direction of the data set on one antenna. This approach is similar to the proposed TRSTC; however, the time-reversal is carried out on the data domain instead of the signal domain and hence requires two separate modulations for each block. In the present proposal, the transmitted signal itself is time-reversed which can be implemented by simply reading memory in reverse order and hence exhibits a lower complexity compared to the work in [11]. Furthermore, the approach in [11] requires long guard intervals due to the long filter tails of FBMC that are used to achieve low $\mathrm{OOB}$ emission and hence reduces spectral efficiency.

In [12], the combination of FBMC/OQAM with circular convolution and CP addition is proposed. This proposal is equivalent to applying standard offset-QAM modulation to GFDM. Moreover, [12] proposes to use timewindows over the blocks to reduce the $\mathrm{OOB}$ emission caused by discontinuities between blocks, which leads to the WCP-COQAM proposal. It is shown that WCPCOQAM achieves lower OOB emission compared to standard non-windowed GFDM. In the present work, we apply the new technique of CR-OQAM to the GFDM system. We present techniques to achieve transmit diversity for both OQAM and CR-OQAM, which is not covered in [12]. Moreover we analyze the impact of guard symbols (GSs) for reducing OOB emission. We show that GS further reduce the OOB emission compared to [12] and do not necessarily reduce spectral efficiency when replaced with pilot or synchronization symbols. Furthermore, a PAPR analysis is carried out for both GFDM/OQAM and GFDM/CR-OQAM.

The remainder of this paper is organized as follows. Section 2 describes the conventional OQAM transceiver, whereas the proposed modification is presented in Section 3. Section 4 describes the application of
CR-OQAM to GFDM, and performance simulations are provided in Section 5. Finally, Section 6 concludes.

\section{Conventional OFDM/OQAM}

In OFDM/OQAM, complex-valued data symbols $c_{k, m}$ are transmitted on $\mathrm{K}$ subcarriers, where the real and imaginary part are offset by $\frac{T}{2}$ where $T$ is the symbol duration. Each symbol is pulse shaped with a symmetric, realvalued pulse shaping filter $g(t)[3,5]$. The transmission equation is given by

$$
\begin{aligned}
x(t)= & \sum_{\substack{k=0 \\
m \in \mathbb{Z}}}^{K-1}\left(c_{k, m}^{\mathrm{R}} g(t-m T)\right. \\
& \left.+j c_{k, m}^{\mathrm{I}} g\left(t-m T-\frac{T}{2}\right)\right) j^{k} w^{k t}
\end{aligned}
$$

where $w=\exp (j 2 \pi F), F=\frac{1}{T}$, and $c_{k, m}^{\mathrm{R}}$ and $c_{k, m}^{\mathrm{I}}$ are the real and imaginary part of $c_{k, m}$, respectively. Note that, due to the factor $j^{k}$, adjacent subcarriers differ by a phase shift of $\frac{\pi}{2}$.

At the receiver, matched filtering is carried out, i.e.,

$$
\begin{aligned}
& \hat{c}_{k, m}^{\mathrm{R}}=\left.\Re\left(x(t) j^{-k} w^{-k t} * g(-t)\right)\right|_{t=m T} \\
& \hat{c}_{k, m}^{\mathrm{I}}=\left.\Im\left(x(t) j^{-k} w^{-k t} * g(-t)\right)\right|_{t=\left(\frac{1}{2}+m\right) T},
\end{aligned}
$$

where convolution $x(t) * y(t)$ is defined as

$$
\left.(x(t) * y(t))\right|_{t=\tau}=\int_{-\infty}^{\infty} x(t) y(\tau-t) d t .
$$

The corresponding block diagram of the OFDM/OQAM transceiver is presented in Fig. 1. For perfect reconstruction, the orthogonality conditions between the $k$ th and $(k+\kappa)$ th subcarrier that are given by ([5], Eq. (13))

$$
\begin{aligned}
& \Re\left\{\left.\left(g(t) j^{-\kappa} w^{-\kappa t} * g(-t)\right)\right|_{t=m T}\right\}=\delta(\kappa, m) \\
& \Re\left\{\left.\left(j g(t) j^{-\kappa} w^{-\kappa t} * g(-t)\right)\right|_{t=\left(\frac{1}{2}+m\right) T}\right\}=0 \\
& \Im\left\{\left.\left(g(t) j^{-\kappa} w^{-\kappa t} * g(-t)\right)\right|_{t=\left(\frac{1}{2}+m\right) T}\right\}=0 \\
& \Im\left\{\left.\left(j g(t) j^{-\kappa} w^{-\kappa t} * g(-t)\right)\right|_{t=m T}\right\}=\delta(\kappa, m)
\end{aligned}
$$

must be fulfilled for all $\kappa, m$. With the relations $\Re\{j a\}=-\Im\{a\}$ and $\Im\{j a\}=\Re\{a\}$, (5) and (6) are equivalent to (8) and (7), respectively. These conditions are in particular fulfilled, when $g(t)$ is a symmetric, half-Nyquist filter with band-limitation $G(f)=0,|f| \geq F$ [2]. The convolution is expressed with the Fourier transform $\mathcal{F}$ by

$$
\begin{aligned}
\left.\left(g(t) w^{-k t} * g(-t)\right)\right|_{t=\tau} & =\mathcal{F}^{-1}\left\{S_{k}(f)\right\}(\tau) \\
& =s_{k}(\tau),
\end{aligned}
$$

where $S_{k}(f)=G(f-k F) G^{*}(f)$ is the spectrum of the intercarrier interference (ICI) from the $k^{\prime}$ th to the $\left(k^{\prime}+\right.$ $k)$ th subcarrier, $s_{k}(t)$ is the corresponding time domain 


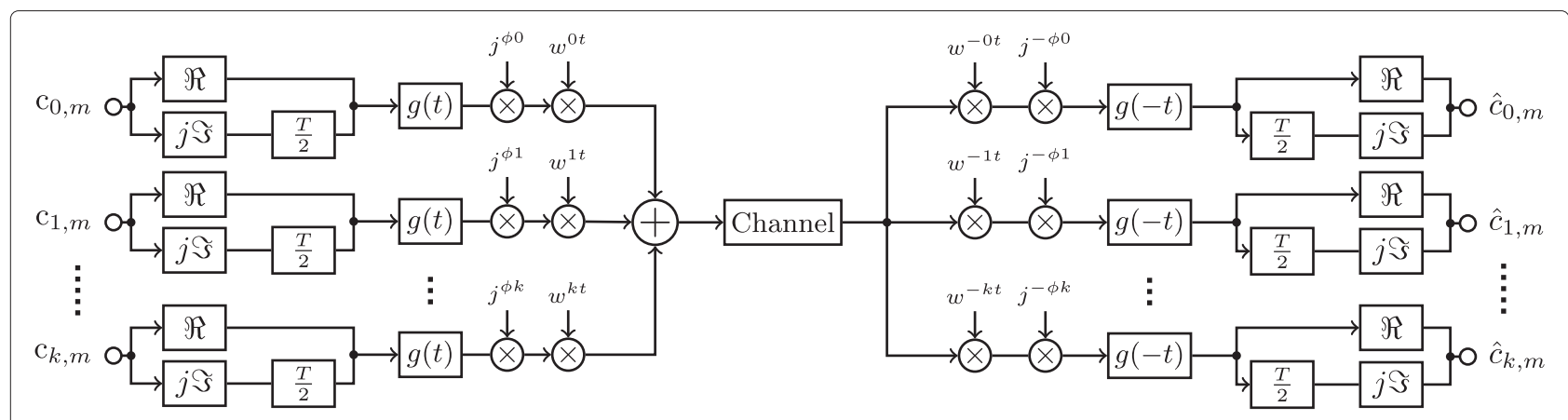

Fig. 1 Block diagram of the OFDM/OQAM $(\phi=1)$ and OFDM/CR-OQAM $(\phi=0)$ transceivers

function, $G(f)$ is the Fourier transform of $g(t)$, and $(\cdot)^{*}$ denotes complex conjugation. Figure 2a depicts $s_{1}(t)$ for a root-raised-cosine (RRC) filter with roll-off $\alpha=0.75^{1}$.

\section{Conjugate root multicarrier OQAM}

In literature, mostly symmetric half-Nyquist filters are employed for MC OQAM systems. We propose the application of non-symmetric CR filters $[13,14]$ for $M C$ OQAM systems.

Assume an even $H(f)$ with band limitation $H(f)=0$ for $|f| \geq F$. Then, $H(f)$ fulfills the 1st Nyquist criterion [13] if

$$
\forall f \in[0, F]: H(f)+H(f-F)=1 .
$$

Its corresponding half-Nyquist filter is given by $G(f)=$ $\sqrt{H(f)}$ where the most prominent example is the symmetric raised-cosine (RC) and RRC pair. The according non-symmetric $\mathrm{CR}$ filter $G^{\mathrm{C}}(f)$ is constructed by

$$
G^{\mathrm{C}}(f)=\left\{\begin{array}{l}
H(f)+j \sqrt{(1-H(f)) H(f)} f \geq 0 \\
H(f)-j \sqrt{(1-H(f)) H(f)} f<0
\end{array}\right.
$$

with impulse response $g^{\mathrm{C}}(t)=\mathcal{F}^{-1}\left\{G^{\mathrm{C}}(f)\right\}$. Note that both $G^{\mathrm{C}}(f)$ and $G^{\mathrm{C}}(f)\left(G^{\mathrm{C}}(f)\right)^{*}$ are Nyquist filters (cf. (10)).
Figure $2 \mathrm{~b}$ shows the $\mathrm{RRC}$ and conjugate $\mathrm{RRC}$ (CRRC) filter response. The ICI $S_{1}^{\mathrm{C}}(f)$ between the adjacent subcarriers with $\mathrm{CR}$ filters equals

$$
\begin{aligned}
S_{1}(f) & =\sqrt{H(f) H(f-F)} \\
S_{1}^{\mathrm{C}}(f) & =G^{\mathrm{C}}(f-F)\left[G^{\mathrm{C}}(f)\right]^{*} \\
& =j S_{1}(f),
\end{aligned}
$$

where (14) follows from (11) and (10). Therefore, since

$$
s_{1}^{\mathrm{C}}(t)=j s_{1}(t),
$$

the real and imaginary part of the ICI is exchanged when using a CR filter compared to its standard half-Nyquist version. Both $S_{1}^{\mathrm{C}}(f)$ and $s_{1}^{\mathrm{C}}(t)$ are presented in Fig. 2c. Comparing Fig. $2 \mathrm{a}$ and $\mathrm{c}$ reveals that the intrinsic interference $s_{0}(t)$ on the same subcarrier is equal for both OQAM and CR-OQAM. However, the interference on the adjacent subcarrier $s_{1}^{C}(t)$ has exchanged real and imaginary parts compared to $s_{1}(t)$. Accordingly, for CR-OQAM, the adjacent subcarrier real and imaginary part of the transmitted symbols should also be exchanged compared to conventional OQAM. This observation leads to the following modified orthogonality conditions and changed phase space.

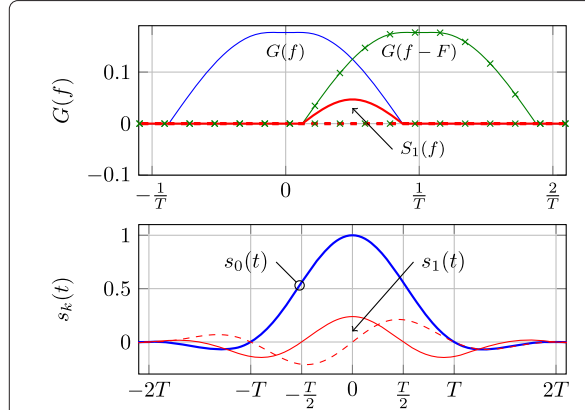

(a) ICI of RRC.
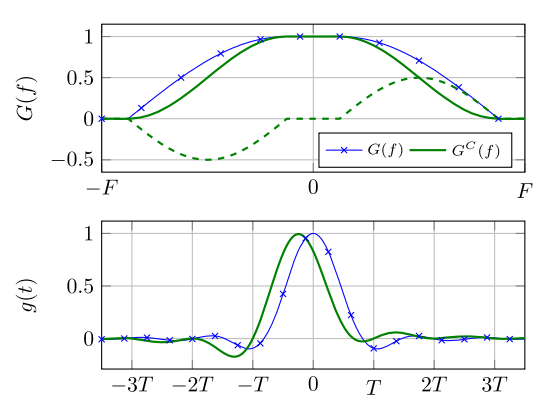

(b) RRC and CRRC impulse and frequency response.
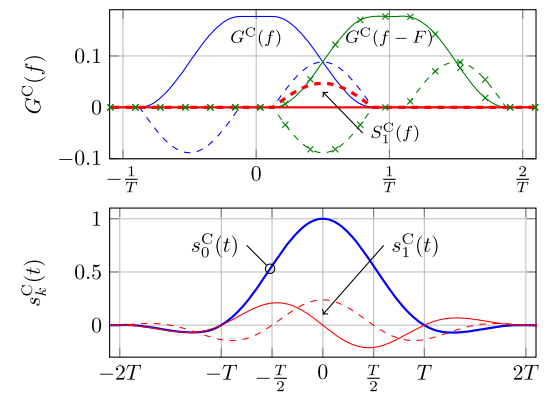

(c) ICI of CRRC.

Fig. 2 Illustration of filters and interference responses. Solid and dashed lines represent the real and imaginary part, respectively. a, $\mathbf{c} I \mathrm{Cl}$ of the adjacent channel and ISI on the same channel for OQAM and CR-OQAM, respectively. b Filter response of RRC and CRRC filters 
If $g(t)$ fulfills (5)-(8), the according $g^{\mathrm{C}}(t)$ fulfills

$$
\begin{aligned}
& \Re\left\{\left.\left(g^{\mathrm{C}}(t) w^{-k t} * g^{\mathrm{C}}(-t)\right)\right|_{t=m T}\right\}=\delta(k, m) \\
& \Re\left\{\left.\left(j g^{\mathrm{C}}(t) w^{-k t} * g^{\mathrm{C}}(-t)\right)\right|_{t=\left(\frac{1}{2}+m\right) T}\right\}=0 .
\end{aligned}
$$

Note, that compared to (5)-(8) the factor $j^{k}$ has been removed. Consequently, an OFDM/CR-OQAM system that uses a CR filter $g^{\mathrm{C}}(t)$ can be described by the modulation equation

$$
\begin{aligned}
x(t)= & \sum_{\substack{k=0 \\
m \in \mathbb{Z}}}^{K-1}\left(c_{k, m}^{\mathrm{R}} g^{\mathrm{C}}(t-m T)\right. \\
& \left.+j c_{k, m}^{\mathrm{I}} g^{\mathrm{C}}\left(t-m T-\frac{T}{2}\right)\right) w^{k t},
\end{aligned}
$$

the demodulation equations

$$
\begin{aligned}
& \hat{c}_{k, m}^{\mathrm{R}}=\left.\Re\left(x(t) w^{-k t} * g^{\mathrm{C}}(-t)\right)\right|_{t=m T} \\
& \hat{c}_{k, m}^{\mathrm{I}}=\left.\Im\left(x(t) w^{-k t} * g^{\mathrm{C}}(-t)\right)\right|_{t=\left(\frac{1}{2}+m\right) T}
\end{aligned}
$$

and the block diagram in Fig. 1 with $\phi=0$.

The time-frequency phase space of the transmitted symbols for OFDM/OQAM and OFDM/CR-OQAM is shown in Fig. 3 where the real and imaginary values are depicted with $\bigcirc$ and $\bullet$, respectively. Due to the missing $\frac{\pi}{2}$ phase shift between subcarriers, the grid is more regular for the CR-OQAM case. Hence, OFDM/CR-OQAM can be seen as two parallel OFDM/PAM systems transmitting with a time offset of $\frac{T}{2}$ and a phase shift of $\frac{\pi}{2}$. Table 1 shows the values of the intrinsic interference after the analysis filter bank when using RRC and CRRC filters for the OFDM/OQAM systems. As shown, on the adjacent channels, the real and imaginary parts of the interference are exchanged due to the use of CR filters. Note that, even though the CR filter itself is non-symmetric, the interference pattern is symmetric in time since $s_{1}^{C}(t)$ is given by the convolution of the CRRC filter and its matched filter, which eventually creates a symmetric pattern.

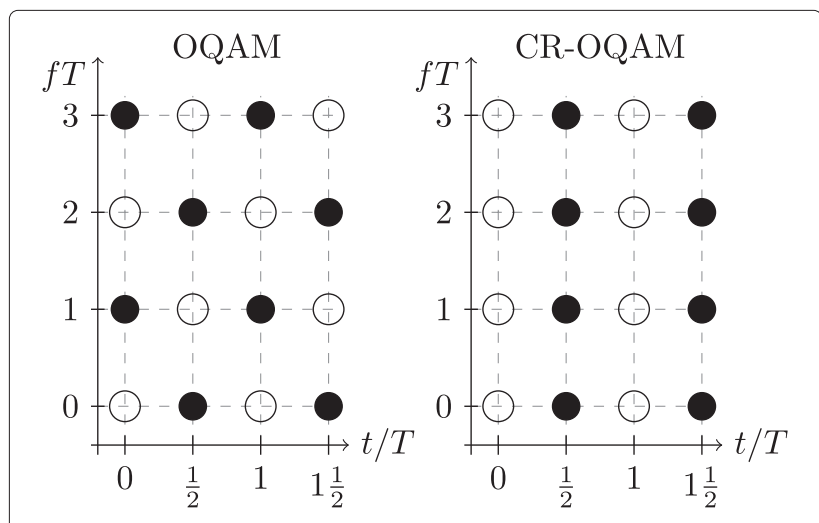

Fig. 3 Time-frequency phase space of OQAM and CR-OQAM
In [6], conjugate OFDM/OQAM is proposed, where forward-time and time-reversed transmit and receive filters alternate along the subcarriers, which is different compared to the present proposal. Using this approach, the symmetry requirement of the prototype filters is relaxed, but still a phase shift between subcarriers is kept, hence keeping the phase space equal to conventional OQAM.

\subsection{Implementation complexity}

In [6], low-complexity formulations for conventional and conjugate OQAM are given. Following a similar argument, the implementation complexity of the present proposal is analyzed and compared with the values from [6], where we consider the number of real-valued arithmetic operations as the figure of merit. Similar to [6], one complex multiplication or addition accounts for six or two real operations, respectively. Let

$$
x_{k, m^{\prime}}= \begin{cases}c_{k, m^{\prime} / 2}^{R} & m \text { even } \\ j c_{k,\left(m^{\prime}-1\right) / 2}^{I} & m \text { odd }\end{cases}
$$

denote the data that is transmitted at the time $m^{\prime} \frac{T}{2}$. Sampling (18) at a frequency $\frac{1}{T}$ and decomposing $t=t_{0}+\frac{n}{T}$ with $t_{0} \in T \mathbb{Z}$, (18) can be reformulated to

$$
x\left(t_{0}+\frac{n}{T}\right)=\sum_{m^{\prime} \in \mathbb{Z}} g^{C}\left(t_{0}+\frac{n}{T}-m^{\prime} \frac{T}{2}\right) P_{n, m^{\prime}}
$$

with

$$
P_{n, m^{\prime}}=\sum_{k=0}^{K-1} x_{k, m^{\prime}} \exp \left(j 2 \pi \frac{k n}{K}\right)
$$

being the K-point inverse discrete Fourier transform (IDFT) of $x_{(\cdot), m^{\prime}}$. Note that the authors in [6] proposed a similar structure as (22), though $P_{n, m^{\prime}}$ is given by an operation involing two $\frac{K}{2}$-point IDFT plus post-processing steps which require in total $\mathcal{C}_{\mathrm{OQAM}}=5 K \log _{2} K+K$ real operations per $T$ seconds [6].

Noting that for the CR-OQAM case, $x_{(\cdot), m^{\prime}}$ is purely real or imaginary for even or odd $m^{\prime}$ respectively, (24) is essentially a real-input IDFT. Hence, with even $m^{\prime}, P_{n, m^{\prime}}$ and $P_{n,\left(m^{\prime}+1\right)}$ can be jointly calculated by a single K-point IDFT of the sequence $c_{(\cdot), m^{\prime} / 2}$ (cf. (21)) by

$$
\begin{aligned}
C_{(\cdot), m} & =\operatorname{IFFT}\left\{c_{(\cdot), m}\right\} \\
P_{k, 2 m} & =C_{k}+C_{K-k}^{*} \\
P_{k, 2 m+1} & =C_{k}-C_{K-k}^{*} .
\end{aligned}
$$

Exploiting the symmetry property for real-input IDFT $P_{k, 2 m}=P_{K-k, 2 m}^{*}$, the operations in (24) require $K$ complex sums and one $K$-point inverse fast Fourier transform (IFFT) which translates to $\mathcal{C}_{\mathrm{CR}}=5 K \log _{2} K-5 K+6$ real operations ${ }^{2}$ every $T$ seconds. 
Table 1 Time-frequency response of OFDM/OQAM and OFDM/CR-OQAM system using RRC and CRRC filters with roll-off $\alpha=1$, respectively

\begin{tabular}{llllllllll}
\hline$t$ & $-2 T$ & $-\frac{3}{2} T$ & $-T$ & $-\frac{1}{2} T$ & 0 & $\frac{1}{2} T$ & $T$ & $\frac{3}{2} T$ \\
\hline$s_{2}(t)$ & 0 & 0 & 0 & 0 & 0 & 0 & 0 & 0 & $2 T$ \\
$s_{1}(t)$ & -0.021 & $-0.000 j$ & -0.106 & $-0.250 j$ & 0.318 & $0.250 j$ & -0.106 & $-0.000 j$ & -0.021 \\
$s_{0}(t)$ & $-0.000 j$ & $-0.000 j$ & $-0.000 j$ & 0.500 & 1.000 & 0.500 & $0.000 j$ & $-0.000 j$ & $0.000 j$ \\
$s_{-1}(t)$ & -0.021 & $0.000 j$ & -0.106 & $0.250 j$ & 0.318 & $-0.250 j$ & -0.106 & $0.000 j$ & -0.021 \\
$s_{-2}(t)$ & 0 & 0 & 0 & 0 & 0 & 0 & 0 & 0 & 0 \\
$s_{2}^{C}(t)$ & 0 & 0 & 0 & 0 & 0 & 0 & 0 & 0 & 0 \\
$s_{1}^{C}(t)$ & $-0.021 j$ & $0.000 j$ & $-0.106 j$ & 0.250 & $0.318 j$ & -0.250 & $-0.106 j$ & $-0.000 j$ & $-0.021 j$ \\
$s_{0}^{C}(t)$ & $-0.000 j$ & $-0.000 j$ & $-0.000 j$ & 0.500 & 1.000 & 0.500 & $0.000 j$ & $-0.000 j$ & $0.000 j$ \\
$s_{-1}^{C}(t)$ & $0.021 j$ & $0.000 j$ & $0.106 j$ & 0.250 & $-0.318 j$ & -0.250 & $0.106 j$ & $-0.000 j$ & $0.021 j$ \\
$s_{-2}^{C}(t)$ & 0 & 0 & 0 & 0 & 0 & 0 & 0 & 0 & 0
\end{tabular}

The subsequent processing of (22) by a polyphase network is identical as in [6] and accounts for equivalent complexity. Accordingly, with the changed phase space of CR-OQAM, the implementation complexity of an OFDM/OQAM scheme can be reduced by $6(K-1)$ real operations per $T$ seconds. Figure 4 shows the number of real operations for the IFFT preprocessing required for OFDM/OQAM according to [6] and the presented OFDM/CR-OQAM. For higher number of subcarriers $K$, the complexity of the proposal converges to the complexity of [6]; however, for a number of subcarriers in the range $2^{4}$ to $2^{11}$, a complexity reduction between 25 and $10 \%$ is achieved.

\section{Application of CR-OQAM in GFDM}

OFDM/OQAM is a streaming-based, filtered MC system, where every symbol overlaps with its adjacent symbols in time. GFDM [8] is a filtered MC system, where circular convolution is applied instead of linear. Hence, its transmit signal exhibits a block structure, and subsequent blocks can be decoupled by a cyclic prefix (CP) to ease equalization.
GFDM is modeled in discrete base band with sampling period $T_{s}$. The transmit signal $\vec{x}$ is given by

$$
\vec{x}=\mathbf{A} \vec{d}
$$

where the columns of the matrix A contain circular timefrequency shifted versions of a prototype transmit filter $g[n]$ with distance $K T_{s}$ in time and $1 /\left(K T_{s}\right)$ in frequency, where $K$ is the number of subcarriers. $\vec{d}$ contains the complex-valued data symbols to be transmitted with the block. By appending a CP, frequency domain channel equalization can be carried out at the receiver, yielding the signal $\hat{\vec{x}}$ and zero-forcing (ZF) or matched filter (MF) detection is applied

$$
\hat{\vec{d}}_{\mathrm{ZF}}=\mathbf{A}^{-1 \hat{\hat{x}}} \quad \hat{\vec{d}}_{\mathrm{MF}}=\mathbf{A}^{\mathrm{H} \hat{\hat{x}}},
$$

where $(\cdot)^{\mathrm{H}}$ denotes the Hermitian conjugate. A main property of GFDM is its good TFL of the transmit filter, which allows to achieve a low $\mathrm{OOB}$ radiation and robustness against asynchronicity [8]. However, when using QAM modulation, the BLT prohibits orthogonality completely, which impacts MF performance while ZF
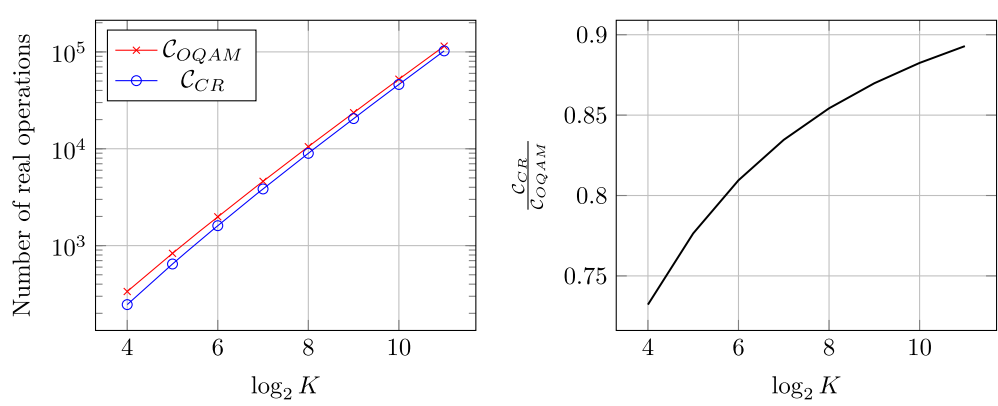

Fig. 4 Comparison of preprocessing complexity of OQAM and CR-OQAM 
detectors introduce noise enhancement and only exist for certain parameter configurations [15]. Hence, with perfect synchronization, SER performance of GFDM is worse compared to an orthogonal system.

To circumvent this problem, OQAM modulation can be applied, which provides orthogonality but still keeps the advantageous property of good time-frequency localization. Looking at the phase space of CR-OQAM (Fig. 3), the GFDM/CR-OQAM modulator is given by

$$
\vec{x}=\mathbf{A} \Re\{\vec{d}\}+j \mathcal{C}_{\frac{K}{2}}(\mathbf{A} \Im\{\vec{d}\}),
$$

where $\mathcal{C}_{u}(\cdot)$ denotes a circular rotation of its argument by $u$ elements. At the receiver, the CR-OQAM detection with the matched filter is simply achieved by

$$
\left.\Re\{\hat{\vec{d}}\}=\Re\left\{\mathbf{A}^{H} \vec{x}\right\}, \quad \Im \hat{\vec{d}}\right\}=\Im\left\{\mathbf{A}^{H} \mathcal{C}_{-\frac{K}{2}}(\vec{x})\right\} .
$$

Time-reversal space-time coding (TR-STC) [16] can be applied to GFDM/CR-OQAM to provide transmit diversity in a fading multipath environment. TR-STC was initially developed for single-carrier systems, where two subsequent time domain codewords are decoupled by a $\mathrm{CP}$ and jointly space-time encoded. For GFDM, one block is treated as a single-carrier codeword and the TR-STC is applied onto two subsequent GFDM blocks [9]. At the receiver, both GFDM blocks are recovered by the TR-STC and processed by the GFDM detector. The approach can be applied to both GFDM and GFDM/(CR-)OQAM, but GFDM/(CR-)OQAM is expected to outperform conventional GFDM due to the kept orthogonality.

\section{Performance evaluation}

The SER performance of GFDM and GFDM/(CR-) OQAM with and without TR-STC has been evaluated in Rayleigh fading multipath channels. The power delay profile of the channel between the transmit and receive antennas is modeled with length 16 where the tap powers decay linearly in $\log$ scale from 0 to $-16 \mathrm{~dB}$. The GFDM parameters are given in Table 2, and the simulation results are presented in Fig. 5, where perfect channel state information was available at the receiver.

The theoretic equations for GFDM/QAM are taken from [9]. Note that as GFDM/(CR-)OQAM is orthogonal,

Table 2 GFDM simulation parameters

\begin{tabular}{lllll}
\hline Parameter & Symbol & QAM & OQAM & CR-OQAM \\
\hline Subsymbol spacing & $K$ & 64 & 64 & 64 \\
Subsymbol count & $M$ & 7 & 7 & 7 \\
Filter & $g[n]$ & RC & RRC & CRRC \\
Filter roll-off & $\alpha$ & 0.5 & 1 & 1 \\
Cyclic prefix & $N_{\text {CP }}$ & 64 & 64 & 64 \\
Detector & & ZF & MF & MF \\
\hline
\end{tabular}

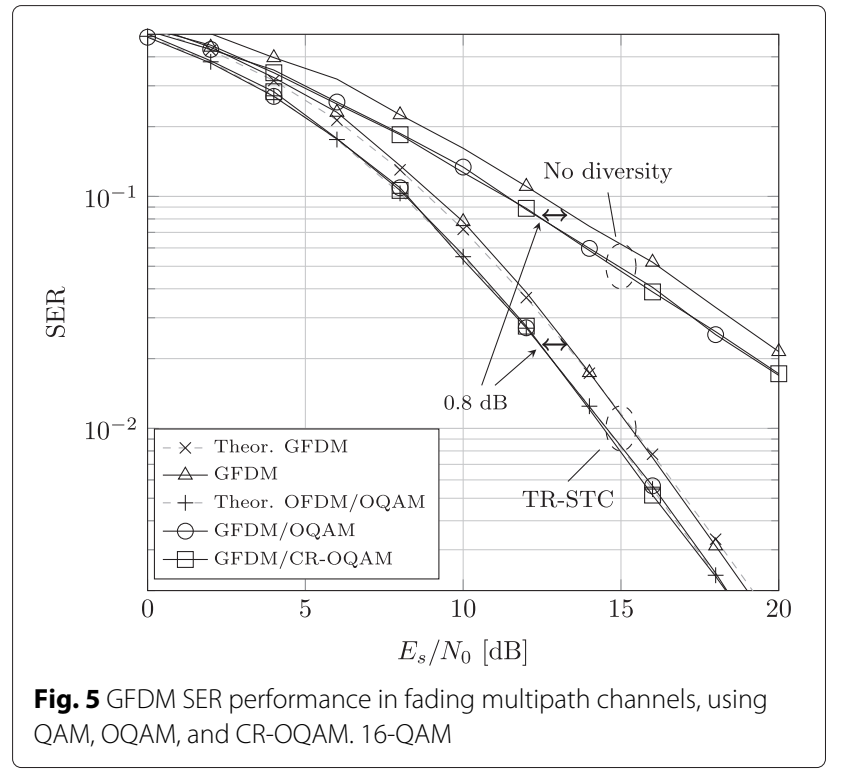

it performs equal to OFDM/OQAM, and the corresponding equations are also taken from ([9], Eq. (19)) with noise-enhancement $\xi=1$.

GFDM/OQAM outperforms conventional GFDM with and without TR-STC due to the orthogonality introduced by the OQAM modulation. The performance gap equals the noise-enhancement of GFDM, which is $0.8 \mathrm{~dB}$ in the presented configuration. Both GFDM and GFDM/(CR-) OQAM show a diversity gain of 2 when combined with TR-STC.

Figure 6 compares the power spectral density (PSD) of GFDM/OQAM and GFDM/CR-OQAM using two GS [8], where the application of CRRC filters significantly reduces the OOB emission. As shown in [8], high OOB emission is mainly due to abrupt signal changes at the GFDM

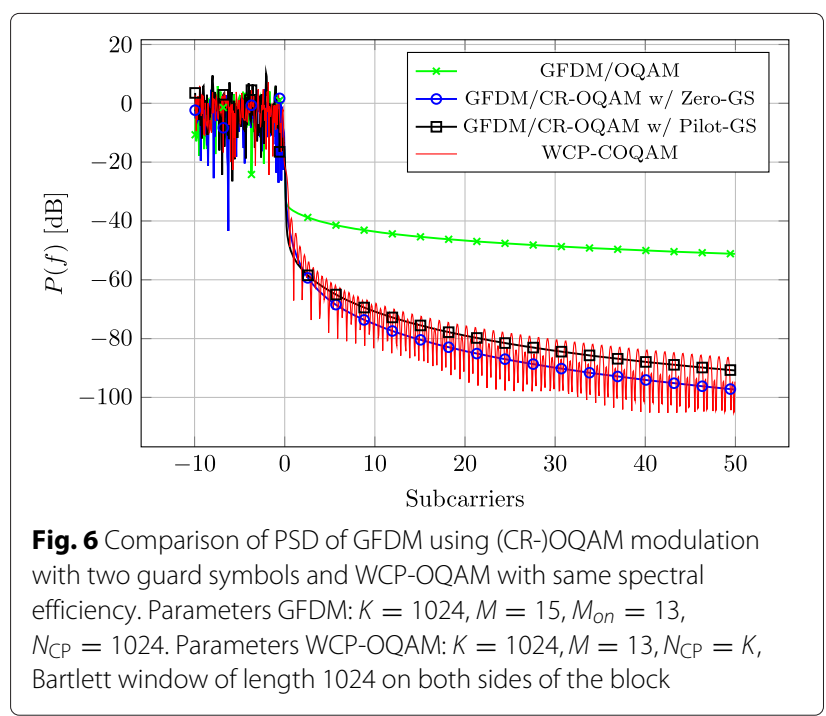


block boundaries. By using GSs with QAM modulation and ISI-free filters, these discontinuities are avoided since the ISI-free property guarantees zeros of the pulse shaping filter at the symbol positions. However, with the introduction of OQAM modulation, also the half-symbol shifts of the pulse shaping filter shall not produce an abrupt signal change and hence the pulse shaping filter is required to vanish at every half-subsymbol time. This particular property is not fulfilled by the RRC filter and hence GS cannot be effectively used with this configuration. On the other hand, the CRRC vanishes at every half subsymbol and hence introduction of GS considerably reduces $\mathrm{OOB}$ emission. Note that the application of GSs requires the $\mathrm{CP}$ to be of length of one complete subsymbol and the empty GSs imply a rate reduction by a factor of

$$
\rho=\frac{M-2}{M+1}
$$

compared to transmission without CP and GSs. However, in order to have a smooth signal transition between subsequent blocks, GSs are not required to be strictly zero but only equal in all blocks. Hence, the resources for GSs can also be used for pilots or synchronization sequences and do not necessarily reduce the system's spectral efficiency. The according spectrum of a GFDM/CR-OQAM system that uses a random GS sequence which is the same for all GFDM blocks is shown in Fig. 6. As shown, the spectrum only slightly degrades compared to the solution employing zero-valued GSs. The present simulation assumes an infinite stream of GFDM blocks. However, in practice, the beginning and end of the stream would need to be smoothened by the use of a window. For comparison, the PSD of the WCP-COQAM proposal [12] is also shown in Fig. 6. WCP-COQAM achieves equal spectral efficiency, by applying a triangular Bartlett window [12] to the signal instead of inserting 2 GSs. As Fig. 6 shows, the PSD of GFDM/CR-OQAM and WCP-COQAM are comparable, but GFDM/CR-OQAM achieves a slightly better OOB emission. Note that the used window is not optimized for minimal OOB emission. On the other hand, the windowed region can hardly be used for pilots or synchronization sequences compared to the GSs approach.

When introducing new waveforms, beneath its OOB emission, also the PAPR is an essential parameter describing the required backoff at the transmitter power amplifier (PA) in order not to introduce non-linear distortions. Figure 7 compares the PAPR of the proposed GFDM/CROQAM with conventional GFDM/OQAM and OFDM, where OFDM uses the same CP length and hence needs more subcarriers in order to achieve equal spectral efficiency. As can be seen, both GFDM/CR-OQAM and GFDM/OQAM have a slightly lower PAPR than OFDM, which is due to the usage of fewer subcarriers and the

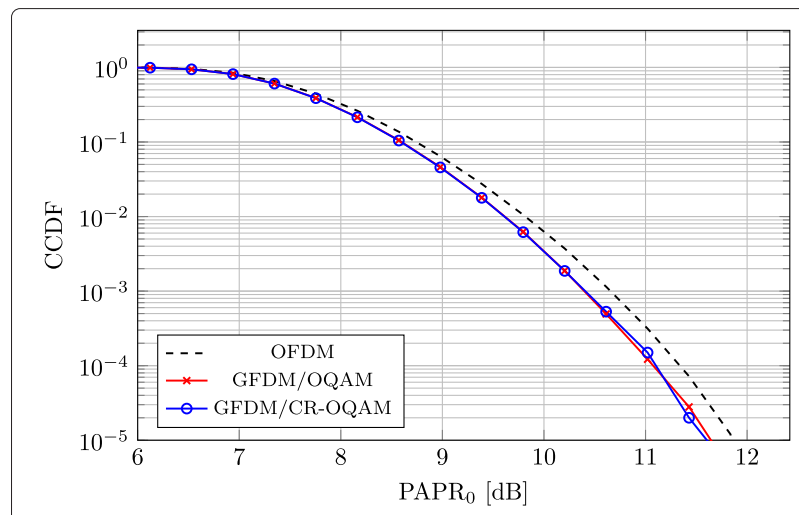

Fig. 7 PAPR comparison of different systems. GFDM parameters from Table 2, OFDM uses 7 . 64 subcarriers, CP length of 64 samples

application of offset-QAM modulation, which can reduce PAPR. Furthermore, the figure shows equal PAPR for both GFDM/OQAM and GFDM/CR-OQAM.

\section{Conclusions}

This paper has presented an alternative approach for the implementation of OQAM when using non-symmetric conjugate root filters. With the proposal, no phase shift between subcarriers is required, making the CR-OQAM time-frequency phase space more regular, and compared to existing work, implementation complexity is reduced. Orthogonality conditions have been stated for the OFDM/CR-OQAM system, and it was proven that CR filters fulfill these. CR-OQAM has been applied to GFDM to create an orthogonal system with good TFL. Space-time code aiming for transmit diversity was applied to GFDM/(CR-)OQAM which outperformed the conventional space-time coded GFDM system. GS were efficiently employed to reduce $\mathrm{OOB}$ emission when using $\mathrm{CR}$ filters, which is not possible with conventional OQAM.

\section{Endnotes}

${ }^{1}$ Note that $\alpha=0.75$ is used for illustration purposes only, because the resulting filter decays slower in the time domain and the assymmetry is more visible. In real implementations, usually full rolloff is considered for OQAM (see Section 5).

${ }^{2}$ Assuming one K-point FFT requires $\frac{K}{2} \log _{2} \frac{K}{4}+1$ complex multiplications and $N \log _{2} N$ complex additions [6].

\section{Competing interests \\ The authors declare that they have no competing interests.}

\section{Acknowledgements}

The work presented in this paper was sponsored by the Federal Ministry of Education and Research within the program "Twenty20 - Partnership for Innoviation" under contract 03ZZ0505B - "fast wireless". The computations were performed at the Center for Information Services and High Performance Computing (ZIH) at TU Dresden. 
Received: 4 August 2015 Accepted: 23 March 2016

Published online: 02 April 2016

\section{References}

1. G Wunder, P Jung, M Kasparick, T Wild, F Schaich, Y Chen, S Brink, I Gaspar, N Michailow, A Festag, L Mendes, N Cassiau, D Ktenas, M Dryjanski, S Pietrzyk, B Eged, P Vago, F Wiedmann, 5GNOW: non-orthogonal, asynchronous waveforms for future mobile applications. IEEE Commun. Mag. 52(2), 97-105 (2014). doi:10.1109/MCOM.2014.6736749

2. B Le Floch, M Alard, C Berrou, Coded orthogonal frequency division multiplex [TV broadcasting]. Proc. IEEE. 83(6), 982-996 (1995). doi:10.1109/5.387096

3. RW Chang, Synthesis of band-limited orthogonal signals for multichannel data transmission. Bell Syst. Tech. J. 45(10), 1775-1796 (1966)

4. JJ Benedetto, C Heil, DF Walnut, in Gabor Analysis and Algorithms, ed. by HG Feichtinger, T Strohmer. Gabor Systems and the Balian-Low Theorem (Birkhäuser, Boston, 1998), pp. 85-122

5. P Siohan, C Siclet, N Lacaille, Analysis and design of OFDM/OQAM systems based on filterbank theory. IEEE Trans. Signal Process. 50(5), 1170-1183 (2002). doi:10.1109/78.995073

6. L Vangelista, N Laurenti, Efficient implementations and alternative architectures for OFDM-OQAM systems. IEEE Trans. Commun. 49(4), 664-675 (2001). doi:10.1109/26.917773

7. K Feher, An asymmetrical pulse shaping technique to combat delay spread. IEEE Trans. Veh. Technol. 42(4), 425-433 (1993). doi:10.1109/25.260770

8. N Michailow, M Matthé, I Gaspar, A Navarro Caldevilla, LL Mendes, A Festag, G Fettweis, Generalized frequency division multiplexing for 5 th generation cellular networks. IEEE Trans. Commun. 62(9), 3045-3061 (2014)

9. M Matthe, LL Mendes, I Gaspar, N Michailow, D Zhang, G Fettweis, Multi-user time-reversal STC-GFDMA for future wireless networks. EURASIP J. Wirel. Commun. Netw. 2015(1), 132 (2015). doi:10.1186/s13638-015-0366-6

10. C Lélé, P Siohan, R Legouable, The Alamouti scheme with CDMA-OFDM/OQAM. EURASIP J. Adv. Signal Process. 2010(1), 1-14 (2010). doi:10.1155/2010/703513

11. M Renfors, T Ihalainen, TH Stitz, in 2010 European Wireless Conference (EW). A block-Alamouti scheme for filter bank based multicarrier transmission (IEEE, 2010), pp. 1031-1037. doi:10.1109/EW.2010.5483517

12. H Lin, P Siohan, Multi-carrier modulation analysis and WCP-COQAM proposal. EURASIP J. Adv. Signal Process. 2014(1), 79 (2014). doi:10.1186/1687-6180-2014-79

13. T Demeechai, Pulse-shaping filters with ISI-free matched and unmatched filter properties. IEEE Trans. Commun. 46(8), 992 (1998). doi:10.1109/26.705392

14. CC Tan, NC Beaulieu, Transmission properties of conjugate-root pulses. IEEE Trans. Commun. 52(4), 553-558 (2004). doi:10.1109/TCOMM.2004.826412

15. M Matthé, LL Mendes, G Fettweis, GFDM in a Gabor transform setting. IEEE Commun. Lett. 18(8), 1379-1382 (2014)

16. N Al-Dhahir, Single-carrier frequency-domain equalization for space-time block-coded transmissions over frequency-selective fading channels. IEEE Commun. Lett. 5(7), 304-306 (2001). doi:10.1109/4234.935750

\section{Submit your manuscript to a SpringerOpen ${ }^{\circ}$ journal and benefit from:}

- Convenient online submission

- Rigorous peer review

- Immediate publication on acceptance

- Open access: articles freely available online

- High visibility within the field

- Retaining the copyright to your article

Submit your next manuscript at $\gg$ springeropen.com 